\title{
Laser Techniques and Laser Physics in Polish Laboratories
}

\author{
Krzysztof M. Abramski \\ Laser and Fibre Electronics Group, Faculty of Electronics, Wrocław University of Technology, \\ Wybrzeże Wyspiańskiego 27, 50-370 Wrocław, Poland,
}

Received March 30, 2014; accepted March 31, 2014; published March 31, 2014

\begin{abstract}
This issue of the Photonics Letters of Poland is a continuation of the overview of Polish research groups working on lasers, their physics and technologies. It contains 16 papers. This set of papers gives a rough scan of the research groups in Poland, which are more or less engaged in the research and development of lasers.
\end{abstract}

The description of a quite original quasi-prototype compact single frequency $\left(\mathrm{Nd}^{3+}: \mathrm{YVO}_{4} / \mathrm{Nd}: \mathrm{YVO}_{4} / \mathrm{KTP}, \quad 532 \mathrm{~nm}\right)$, microchip green solid-state laser with high passive frequency stabilization on a level of $10^{-8}$ over $1 \mathrm{~s}$, as a potential source of metrological applications is presented by Jaroslaw Sotor et al. (Paper No. 1) from Laser and Fiber Electronics Group, Wrocław University of Technology.

Recent achievements (over $11 \mathrm{~W}$ output power) in Qswitching of a very attractive solid state diode pumped Ho:YLF (around 2050nm) laser are presented by J. Kwiatkowski et al. (Paper No. 2) from the group of Prof. Jan Jabczyński, the Institute of Optolectronics, Military Academy of Technology.

The femtosecond fiber CPA (Chirped Pulse Amplification) system based on Yb:KYW crystal developed in the research group of Prof. Czesław Radzewicz of the Institute of Physics from the Polish Academy of Science is described in Paper No. 3, written by Michał Laskownicki et al..

An important achievement in quantum wells technology development and construction of saturable absorber mirrors (SAM) for $\mathrm{Yb}: \mathrm{KYW}$ mode-locked femtosecond lasers was developed by two research groups: from Physics Faculty of Warsaw University and the Institute of Electron Technology in Warsaw (Paper No. 4, by Łukasz J. Zinkiewicz et al.).

The next paper by T. Fok et al. (No. 5) is devoted to extreme ultraviolet and high-order harmonic generation in an extreme ultraviolet region by the so-called "multi-jet gas puff target" technique. These technologies are effectively being developed in the research group of Prof. Henryk Fiedorowicz at the Institute of Optoelectronics, Miltary University of Technology in Warsaw.

A clear description of a two-mode He-Ne laser with an original method of frequency stabilization, by a specially adapted liquid crystal cell, is given in Paper No. 6 (by Grzegorz Budzyń et al. from the Wroclaw University of Technology). The authors applied this laser into a commercial version of laser interferometers.

Paper No. 7 (by Paweł Kaczmarek et al., the Laser and Fiber Electronics Group, Wrocław University of Technology shows a quasi-commercial version of a $\mathrm{CW}$ fiber $1550 \mathrm{~nm}$ laser with an output power of $5 \mathrm{~W}$, built in all-fiber technology, with total controlling of its all parameters.
Well developed high power diode lasers, particularly high power laser arrays, require effective cooling systems. A new solution of miniaturized micro-channel cooling technology is presented by Anna Kozłowska et al. (Institute of Electronic Materials Technology, Warsaw) in Paper No. 8.

Two papers from the research group lead by Prof. Włodzimierz Nakwaski, Łódź University of Technology, are devoted to the modelling of semiconductor laser structures. The first one (Paper No. 9, by Tomasz Czyszanowski et al.) deals with the modelling of new structures for single-mode emission enhancement in a VCSEL structure. The second paper from the same group (Paper No. 10, by Robert Szarzała and W. Nakwaski) introduces a so-called inverted-relief cavity in VCSELs for suppressing higher-order transverse modes.

An important branch of Polish diode lasers research is concentrated around the TopGun company collaborating with the Institute of High Pressure Physics, Ammono Company and Łódź University, where over the last twenty years nitride-based blue diode lasers and superluminescent diodes have been successfully developed. A short overview of recent new concepts (laser diode waveguides, laser arrays, new high power lasers) is presented in Paper No. 11 by the group leaders - Prof. Piotr Perlin and Tadeusz Suski and their co-workers.

Paper No. 12 (by Sławomir Drobczyński et al.) deals more with the applications of lasers. Three types of laser diodes were applied to very original techniques of holographic optical tweezers. Quite successful results of trapping living cells are demonstrated. The research is led by Prof. Jan Masajada, the Institute of Physics, Wrocław University of Technology.

Paper No. 13 (by Debolina Brahma et al.) is not a "Polish paper", but because of the subject, it is included in this edition as a regular paper. It deals with an erbium doped fiber amplifier with a specially designed two-channel configuration in order to obtain flat and wide-band gain characteristics.

Paper No. 14 (by M. Piszczek et al., the Institute of Optoelectronics, Military University of Technology) introduces the idea and basic experiments on a so-called laser photography camera.

The original configuration of a dual-channel Nd:YAG laser with Q-switching specially adapted for direct interference lithography, as a very interesting source for laser micromachining is described in Paper No. 15 by the group leader, Prof. Jan Marczak from the Institute of Optolectronics, Military University of Technology

A phosphate photonic Ytterbium doped fiber laser based on designed and realised photonic crystal fiber at the Institute of Electronic Materials Technology in Warsaw, with an over 10W output power is described in Paper No.16 by Marcin Fronczyk, from the research group led by Prof. Ryszard Buczyński. 